\title{
Antithrombotic Management in Ischemic Stroke with Essential Thrombocythemia: Current Evidence and Dilemmas
}

\author{
Shubhabrata Das ${ }^{a}$ Anasua Deb ${ }^{b}$ Tanmoy Pal ${ }^{c}$ \\ aDepartment of Medicine, University of Toronto, Toronto, ON, Canada; ${ }^{\mathrm{b}}$ Department of Internal Medicine, Texas \\ Tech University Health Sciences Center, Lubbock, TX, USA; 'Department of Neurology, Neotia Getwel Healthcare \\ Centre, Siliguri, India
}

\section{Highlights of the Study}

- A high index of suspicion is needed for essential thrombocythemia in patients with ischemic stroke presenting with high platelet counts.

- Low-dose aspirin twice daily is recommended for patients with atherothrombotic ischemic stroke with essential thrombocythemia in presence of risk factors such as age $>60$ years, Janus kinase 2 mutation, or presence of cardiovascular risk factors.

- Dilemmas exist for antithrombotic management in ischemic stroke associated with atrial fibrillation or cerebral venous thrombosis in presence of the same risk factors.

\section{Keywords}

Essential thrombocythemia - Ischemic stroke ·

Antithrombotic management · Low-dose aspirin

\section{Abstract}

Thrombotic diseases like ischemic stroke are common complications of essential thrombocythemia (ET) due to abnormal megakaryopoiesis and platelet dysfunction. Ischemic stroke in ET can occur as a result of both cerebral arterial and venous thrombosis. Management of ET is aimed at preventing vascular complications including thrombosis. Acute management of ischemic stroke in ET is the same as that in the general population without myeloproliferative disorders. However, an ET patient with ischemic stroke is at high risk for rethrombosis and is therefore additionally managed with cytoreductive therapy and antithrombotic agents. Given abnormal platelet production in ET, there is suboptimal suppression of platelets with the standard recommended dose of aspirin for cardiovascular (CV) prevention. Hence, for optimal CV protection in ET, low-dose aspirin is recommended twice daily in an arterial thrombotic disease like atherothrombotic ischemic stroke in presence of the following risk factors: age $>60$ years, Janus kinase 2 V617F gene mutation, and presence of CV risk factors. In the presence of the same risk factors, concurrent antiplatelet and anticoagulant therapy is suggested for venous thrombosis. However, increased risk of bleeding with dual antithrombotic agents poses a significant challenge in their use in cerebral venous thromboembolism or atrial fibrillation in presence of the above-mentioned risk factors. We discuss these dilemmas regarding antithrombotic management in ischemic stroke in ET in this case-based review of literature in the light of current evidence.

(C) 2021 The Author(s)

Published by S. Karger AG, Basel karger@karger.com www.karger.com/mpp

Karger $\stackrel{\text { ' }}{5}$

BOPEN ACCESS
(C) 2021 The Author(s)

Published by S. Karger AG, Basel

This is an Open Access article licensed under the Creative Commons Attribution-NonCommercial-4.0 International License (CC BY-NC) (http://www.karger.com/Services/OpenAccessLicense), applicable to the online version of the article only. Usage and distribution for commercial purposes requires written permission.
Correspondence to:

Shubhabrata Das, shubhabrata.das@mail.utoronto.ca 


\section{Introduction}

Myeloproliferative neoplasm (MPN) is an umbrella term consisting of clonal stem cell disorders such as essential thrombocythemia (ET), polycythemia vera (PV), primary myelofibrosis (PMF), and chronic myeloid leukemia. ET is the most common type of BCR-ABL-negative MPN with an incidence and prevalence of $1-2.5$ cases/100,000 population/year and $38-57 / 100,000$ population in the USA, respectively [1]. Importantly, ET, PV, and PMF share 3 mutually exclusive driver mutations in the Janus kinase 2 (JAK2), calreticulin (CALR), and myeloproliferative leukemia virus (MPL) genes [2]. Mutation in JAK2 is the most common genetic mutation found in $~ 99 \%$ of PV patients and in $50-65 \%$ of ET and PMF patients. The prevalence of CALR and MPL genetic mutation in ET and PMF is around $15-30 \%$ and $4-8 \%$, respectively [3]. An important step in the diagnosis of ET is to rule out causes of reactive thrombocytosis such as iron deficiency, blood loss, infection, inflammation, postsplenectomy, and malignancy [4]. Apart from ET, common causes of clonal thrombocytosis include early as well as prefibrotic variant PMF and PV. The World Health Organization (WHO) classification system defining major and minor criteria helps the diagnosis of ET from other specific MPNs (see Table 1) [5].

The prognosis of ET patients is slightly better than other BCR-ABL-negative MPNs with a median estimate of survival of $\sim 20$ years as compared to $\sim 14$ and $\sim 6$ years for PV and PMF, respectively [6]. The higher mortality in patients with PV as compared to patients with ET is primarily driven by higher leukemic transformation $(\sim 6-$ $19 \%$ vs. $2-5 \%$ at 15 years) and increased fibrotic progression (6-14\% vs. $4-11 \%$ at 15 years) in this subgroup of MPNs [7]. To date, there has not been any disease-modifying treatment for ET preventing leukemic or fibrotic transformations or prolonging survival. Current goals of management of ET include alleviation of symptoms and minimizing bleeding and thrombotic complications in high-risk patients [8]. Recognizing the microvascular symptoms is important, as one-half of patients with ET are discovered incidentally with high platelet counts in complete blood count ordered for some other reasons. Microvascular vasomotor symptoms have been reported in $10-40 \%$ of patients [9-12] which include headache, syncopal/presyncopal events, atypical chest pain, paresthesia, livedo reticularis, erythromelalgia, transient neurological symptoms like amaurosis fugax, and migraine. These microvascular symptoms are thought to be caused by thrombosis in microvasculature as a result of throm- boxane-related platelet activation and platelet dysfunction in ET. Macrovascular complications including thrombosis and bleeding have been reported in $10-20 \%$ and $5-40 \%$ ET patients, respectively [10-15]. Such thrombotic complications are significant causes of morbidity, and therefore management options include risk stratification identifying those at increased risk of thrombotic events. Cerebrovascular thrombotic events are common in these patients and likely to occur as a result of cerebral arterial and venous thrombosis [16-19]. Abnormal megakaryopoiesis puts these patients at increased risk of cardiovascular $(\mathrm{CV})$ diseases beyond conventional $\mathrm{CV}$ risk factors. Hence, antithrombotic management in ET patients with ischemic stroke or transient ischemic attack (TIA) is challenging as these patients might require further optimization beyond conventional guideline-directed CV risk factor prevention/management. In this casebased review, we explore the epidemiology of ischemic stroke in ET and the current dilemmas on antithrombotic management in this condition.

\section{Case Report}

A 64-year-old male presented to the hospital with a 2-day history of vertigo, ataxic gait, vomiting, hoarseness of voice, and nasal regurgitation. On examination, he was alert, conscious, and oriented with stable vital signs. He had gaze-evoked horizontal nystagmus with a rotatory component on right gaze, reduced movement of the soft palate, absent gag reflex, and tongue deviated toward the right side. He also had oropharyngeal weakness on assessment of swallowing. The rest of the cranial nerve examination was normal including pupillary, extraocular movement, and facial features. Although there was pronator drift, the motor power was preserved in the left upper limb. There was no motor deficit in the right side of the body. Babinski sign was positive on the left side. Deep tendon reflexes in all 4 limbs were normal. Pain sensation was uniformly reduced over the left half of the body excluding the face with preservation of other sensations.

MRI of the brain revealed acute infarct involving the lateral part of medulla and pons on the right side (Fig. 1). Due to his late presentation, he was not a candidate for any revascularization interventions. On day 2 of admission, his motor neurodeficit further progressed on the left side of the body with a decreased level of consciousness. Repeat MRI brain revealed further extension of diffusion restriction to the whole of the right half of pons and medulla (Fig. 2). MR angiography of the brain showed narrowing of the caliber of the right vertebral artery. He was admitted to ICU for neurovital monitoring. After 5 days of ICU stay, he was medically stable and was transferred to stroke rehabilitation. After ruling out hemorrhage in repeat brain imaging, aspirin $75 \mathrm{mg}$ daily was started.

Further evaluation for the risk factors of stroke revealed a current history of smoking and hypertension for 10 years controlled with antihypertensive medications. Continuous cardiac monitoring for $48 \mathrm{~h}$ was negative for atrial fibrillation (AF). His complete 


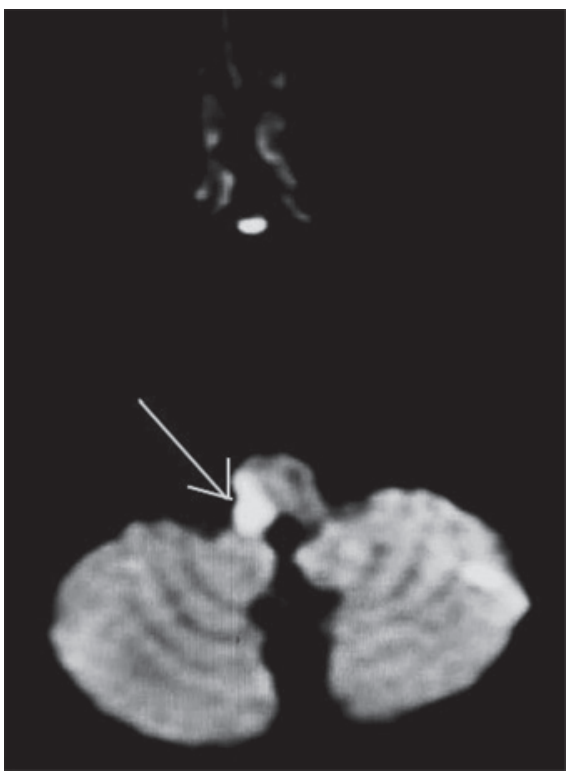

Fig. 1. MRI (DWI) brain showing acute infarct involving the lateral part of medulla and pons on the right side. DWI, diffusion weighted imaging.

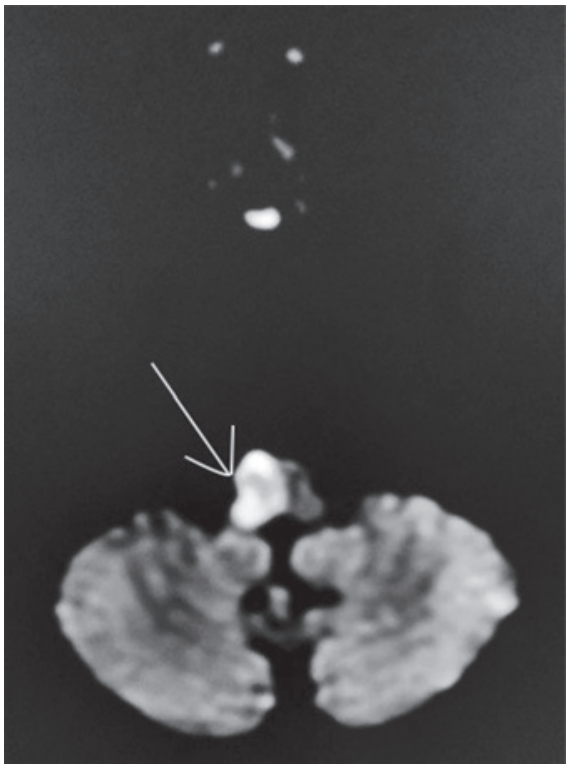

Fig. 2. MRI brain (DWI) showing further extension of the infarct on the next day as compared to Figure 1. DWI, diffusion weighted imaging.

Table 1. World Health Organization criteria for ET [5]

\section{Major criteria}

Platelet count $\geq 450 \times 10^{9} / \mathrm{L}$

Bone marrow biopsy showing proliferation mainly of the megakaryocyte lineage with increased numbers of enlarged, mature megakaryocytes with hyperlobulated nuclei. No significant increase or left shift in neutrophil granulopoiesis or erythropoiesis and very rarely minor (grade 1) increase in reticulin fibers

Not meeting WHO criteria for BCR-ABL1 + CML, PV, PMF, myelodysplastic syndromes, or other myeloid neoplasms

Presence of JAK2, CALR, or MPL mutation

\section{Minor criterion}

Presence of a clonal marker or absence of evidence for reactive thrombocytosis

Diagnosis of ET requires meeting all 4 major criteria or the first 3 major criteria and the minor criterion. CML, chronic myeloid leukemia; ET, essential thrombocythemia; PMF, primary myelofibrosis; PV, polycythemia vera.

blood count was abnormal with platelet count $980,000 / \mathrm{cmm}$, total leukocyte counts $11,900 / \mathrm{cmm}$, and hemoglobin $13.5 \mathrm{~g} / \mathrm{dL}$. His platelet count was found to be persistently elevated, and peripheral blood smear showed presence of thrombocytosis without any blasts or dysplastic cells. No abnormality was detected in other laboratory results. Common causes of reactive thrombocytosis like infection, blood loss, iron deficiency, and trauma were ruled out. Genetic markers of clonal thrombocytosis were studied; exon 9 mutation of the calreticulin (CALR) gene without any mutation of Janus kinase (JAK)2V617F and myeloproliferative leukemia virus (MPL) genes was noted. Furthermore, the BCR-ABL1 gene was not detected by reverse transcriptase polymerase chain reaction. Later after discharge, the patient underwent a bone marrow biopsy, which revealed hypercellular marrow with normoblastic erythropoiesis, myelopoiesis showing maturation up to neutrophils, and megakaryocytic hyperplasia with slight variation in the size of megakaryocytes and mostly normal morphology. He was diagnosed with ET per WHO diagnostic criteria and was referred to hematology services for further management. His antithrombotic management was further optimized with twice daily lowdose aspirin according to evidence-based management; however, he was lost to further follow-up.

\section{Discussion}

We reviewed the existing literature on ischemic stroke in ET focusing on epidemiology, risk factors, and dilemmas on antithrombotic management according to the current evidence. 


\section{Epidemiology of Ischemic Stroke in ET}

We conducted a literature search on ischemic stroke in ET in the PubMed database using the medical subject headings (MESH) "Essential Thrombocythemia" and "Stroke." The initial search result generated a total of 30 articles. Of these, only 3 were case series or studies, while the rest were either case reports or published in languages other than English or not focused on stroke.

In a retrospective analysis of 102 patients with ET followed up for 5 years, Posfai et al. [18] found that 11 patients ( $n=4$ males and 7 females) with a median age of 67 years developed one or more ischemic strokes. At the time of the stroke, 8 out of these 11 cases were already on antiplatelet therapy, alone or in combination with cytoreductive therapy. All patients had CV risk factors with 9 patients having at least 2 risk factors and 3 patients having a history of a cerebrovascular event prior to the diagnosis of ET. Most of these were lacunar strokes or associated with white mater chronic microangiopathic changes as evidenced by brain imaging. Another case series by Kato et al. [17] reported that out of 10 patients presenting with stroke in ET ( $n=3$ males and 7 females, mean age $65 \pm 21$ years), 8 patients had atherosclerotic risk factors. In fact, ischemic stroke was the first manifestation of ET in 8 patients. A total of 13 acute cerebrovascular events following diagnosis of ET were reported in this study, 4 of which were TIAs and 9 were cerebral infarctions (3 with watershed-type infarcts without large artery stenosis and 1 with posterior circulation stroke). Five of these patients had a previous history of ischemic stroke before the diagnosis of ET and were on antiplatelet medication prior to recurrent strokes; 3 of them were on both antiplatelet and cytoreductive therapies [17]. Richard et al. [19] retrospectively studied 14 ischemic stroke patients with ET ( 9 women and 5 men), aged 40-87 years, including 2 patients presenting with internal carotid artery thrombosis. Twelve of these 14 patients had atherosclerotic risk factors, and 1 patient was already on antithrombotic therapy. Diagnosis of ET was made only after the onset of stroke in 12 out of 14 patients [19]. These studies demonstrate that ET increases the risk of ischemic stroke which is further augmented by conventional CV risk factors. Moreover, most ischemic strokes in these patient populations were of anterior circulation involvement, in contrast to our patient, who presented with a posterior circulation stroke. Since many of these reported cases were recurrent strokes developing even when patients were taking antiplatelet medications, it is important to ascertain whether there is a need to further optimize antithrombotic management beyond the current recommendation for $\mathrm{CV}$ prevention.

\section{Thrombotic Risk Estimation in ET}

Historically, the European Leukemia Network risk stratification in ET has included a 2-tiered system which classified these patients into low-risk and high-risk categories based on the respective absence or presence of age $>60$ years or history of thrombosis [20]. Over the years, ET prognostication systems have evolved. Thrombotic risk in ET is calculated according to the results of the study by Barbui et al. [21] aimed at developing and validating a prognostic score for thrombosis in patients with WHO-defined ET. According to this study, age $>60$ years (hazard ratio $[\mathrm{HR}]=1.5$ ), JAK2 V617F mutation $(\mathrm{HR}=2)$, history of thrombosis $(\mathrm{HR}=1.9)$, and $\mathrm{CV}$ risk factors such as hypertension, hypercholesterolemia, diabetes mellitus, and smoking $(\mathrm{HR}=1.6)$ are independent risk factors for thrombosis in this patient demographic. A new prognostic model, International Prognostic Score for Thrombosis in Essential Thrombocythemia (IPSETthrombosis), was developed which incorporated each of these risk factors giving an integer weight close to the HR (e.g., 2 points for JAK2 V617F mutation, 2 points for a prior history of thrombosis, 1 point for age $>60$ years, and 1 point for $\mathrm{CV}$ risk factors). This IPSET-thrombosis score can be calculated using an online calculator [22]. A score of $0-1$ points indicates low risk, 2 points indicate intermediate risk, and 3 points or more high risk [21]. The incidence of thrombosis per year was 1.0, 2.4, and $3.6 \%$ for low-risk, intermediate-risk, and high-risk categories, respectively. The original IPSET risk stratification was further revised based on the observation that JAK2V617F and CV risk factors are independent risk factors for thrombosis. The new risk stratification includes 4 categories: very low risk (no thrombosis history, age $\leq 60$ years, and JAK2 unmutated), low risk (no history of thrombosis, age $\leq 60$ years, and JAK2 mutated), intermediate risk (no history of thrombosis, age $>60$ years, and JAK2 unmutated), and high risk (thrombosis history or age $>60$ years and JAK2 mutated). Incidence of thrombosis per year for very low-risk, low-risk, intermediate-risk, and high-risk categories without any CV risk factors was estimated as $0.4,1.6,1.4$, and $2.4 \%$, respectively. The presence of CV risk factors increased the incidence in each of these groups, with estimates of 1.1, $2.6,1.6$, and $4.2 \%$, respectively [23]. The revised IPSET model was further validated in a study with $585 \mathrm{ET}$ patients which reported that only JAK2V617F (HR: 1.8, 95\% CI: 1.1-2.9) and history of thrombosis (HR: 2.1, 95\% CI: 1.2-3.6) were independent predictors of future thrombotic events [24]. 


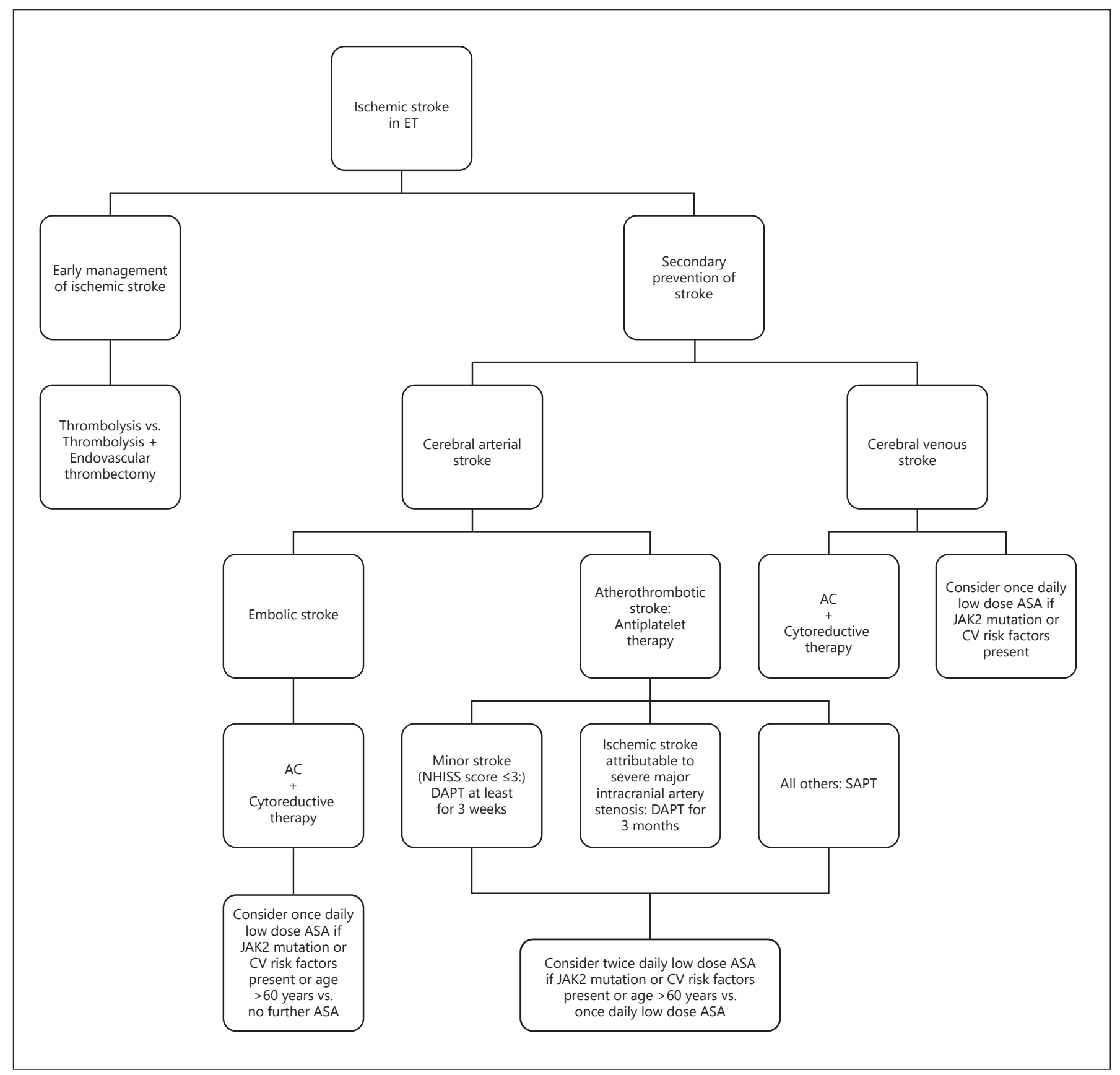

Fig. 3. An approach to antithrombotic management of ischemic stroke in ET. AC, anticoagulation; ASA, aspirin; $\mathrm{CV}$, cardiovascular; DAPT, dual antiplatelet therapy; JAK2, Janus kinase2 gene; SAPT, single antiplatelet therapy; ET, essential thrombocythemia.

Other Emerging Risk Factors of Thrombosis in ET

Leukocytosis

In addition to the risk factors discussed above, leukocytosis has been reported as a risk factor of thrombosis, mainly arterial thrombosis. One of the first studies to demonstrate this was a study that validated the IPSET- thrombosis model in 891 ET patients. In this study, the authors showed that white blood cell (WBC) count $>11,000 / \mathrm{cmm}$ was a risk factor for arterial thrombosis but not for venous thrombosis [25]. Several other studies have found that an increased WBC count at diagnosis is associated with an increased risk of subsequent thrombo- 
sis $[26,27]$. The exact mechanism of increased risk of thrombosis associated with leukocytosis in ET is not known. The European Collaboration on Low-Dose Aspirin in Polycythemia Vera (ECLAP) study also found similarly increased risk of thrombosis associated with leukocytosis in PV patients, particularly for arterial thrombosis such as myocardial infarction [28]. On the contrary, some studies did not find any association between leukocytosis and increased thrombotic risk in ET patients, however associated with worse long-term survival $[29,30]$. A recent meta-analysis involving $>30,000$ patients with ET and PV concluded that the relative risk of thrombosis was 1.59 (95\% CI: 1.4-1.8) in the presence of leukocytosis, mainly driven by ET $(1.7 ; 1.4-1.9)$ and arterial thrombosis $(1.5 ; 1.1-1.9)$ [31]. In light of these pieces of evidence, further research is needed to evaluate whether leukocytosis should be another target to treat for effective reduction of risk of thrombosis in ET as some of the cytoreductive therapy targets WBC while others do not $[32,33]$.

\section{Calreticulin Gene}

A mutation in the Calreticulin (CALR) gene was seen in $20-25 \%$ of ET patients as compared to JAK2 mutation which is seen in $55-60 \%$ of ET patients $[34,35]$. Overall, CALR mutation is associated with a lower incidence of thrombosis than JAK2 mutation. In a prospective cohort study of 894 ET patients, the cumulative 10-year incidence of thrombosis with CALR mutation $(11.0 \%$; $95 \%$ CI: 6.3-17.1) was lower than those with JAK2 mutation (21.0\%; 95\% CI: 16.6-25.7) [34]. A similar observation of lower incidence of thrombosis ( 1.3 vs. $2.0 \%$ patient year; HR 0.6; 95\% CI: 0.3-1.1) was found in another cohort of ET patients $(n=1,150)$ followed up for 4.1 years where the incidence of CALR mutation was $14 \%$ versus $64 \%$ with JAK2 mutation. Ninety-six percent of those with CALR mutation comprised individuals with a low and intermediate risk of thrombosis according to the IPSET model. However, in multivariate models, CALR mutation did not show any association with increased risk of thrombosis suggesting that CALR mutation status does not have a significant impact on the IPSET-thrombosis prognostic score [36]. Another cohort of 576 ET patients, with a median follow-up period of 6 years, had similar findings (major thromboses $13.5 \%$ in CALR mutation vs. $30.1 \%$ in JAK2 mutation) [37]. The lower incidence of thrombosis is likely due to lower platelet activation in CALR-mutated ET patients than those with JAK2 mutation as reported in a platelet function study [38]. In the light of these pieces of evidence, CALR mutation is not included in prognostication of ET patients [21, 22, 39]. Interestingly, our

Ischemic Stroke in Essential

Thrombocythemia patient presented with ischemic stroke and had CALR mutation, in addition to the risk factors of age $>60$ years and hypertension.

\section{Platelet Count}

In a study validating the IPSET-thrombosis model, platelet count $>1,000,000 / \mathrm{cmm}$ was associated with a lower risk of arterial thrombosis (HR 0.4 [0.2-0.8], $p=$ 0.007 ) as well as overall thrombotic risk (HR 0.5 [0.3-0.8], $p=0.009$ ) [25]. Similarly, a multicenter cohort study of ET patients $(n=1,201)$ followed for about 8 years concluded that lower platelet counts at diagnosis of ET were predictive of worse thrombosis-free survival $(p=0.03)$. Moreover, a baseline platelet count of $944,000 / \mathrm{cmm}$ had the best predictive threshold according to receiver operating characteristic analyses based on thrombotic events during follow-up [40]. There is no definite explanation for this apparently counterintuitive association between platelet count and risk of thrombosis. Whether acquired von Willebrand disease (aVWD) which is associated with bleeding and have been reported with high platelet count in ET actually protects from thrombotic disease needs to be explored further [41].

\section{Management of Ischemic Stroke with ET}

Management of ischemic stroke in patients with ET includes general management of acute ischemic stroke, secondary prevention, and cytoreductive therapy in high-risk patients. Based on the current evidence [42, 43], we presented a general approach to antithrombotic management of ischemic stroke in ET in Figure 3. The basis of antithrombotic management in ET is derived from clinical trials of antiplatelet use in PV. Moreover, based on expert opinion, dosing and dose schedule of antiplatelet medications in ET patients with thrombotic disease is different from conventional dosing used for $\mathrm{CV}$ protection. In the next few paragraphs, we discuss these evidence and dilemmas.

\section{Antiplatelet Use in ET}

Aspirin is recommended in any category of ET patients with CV risk factors, JAK2V617F mutation, age $>60$ years, or a history of thrombosis [42]. In the absence of evidence from clinical trials for evaluation of hard clinical endpoints, this recommendation of use of ASA in ET was derived from its use in PV to prevent thrombotic complications $[44,45]$. As the risk of thrombosis in the very low-risk category is comparable to the general nonMPN population, the benefit of using aspirin in this subgroup is uncertain and should be avoided in favor of watchful monitoring and observation [46]. 
Low-Dose Aspirin Once Daily versus Twice Daily

Although aspirin was useful in preventing thrombotic complications in $\mathrm{PV}$, there is evidence of inadequate suppression of platelets at the dosing intervals uniformly recommended for prevention of $\mathrm{CV}$ disease. This is due to altered pharmacodynamics in ET patients with abnormal megakaryopoiesis. An increase in immature platelets results in a shorter duration of action of lowdose aspirin through faster renewal of cyclooxygenase enzyme as evidenced by increase in platelet activation markers (thromboxane metabolites). This was not corrected by increasing the daily dose of aspirin (200 vs. 100 $\mathrm{mg}$ ) given once daily [47-49]. Based on these pieces of evidence, the current expert recommendation is to use low dose (81 mg).

Aspirin twice daily is recommended in cases of (1) high-risk category arterial thrombotic disease (like atherothrombotic ischemic stroke) with either of these 3 risk factors: age $>60$ years or JAK 2 mutation or presence of $\mathrm{CV}$ risk factors; (2) intermediate-risk category with $\mathrm{CV}$ risk factors; and (3) low-risk patients with CV risk factors (Fig. 3) [39, 43]. Although platelet count is not included for risk estimation, aVWD is associated with ET and was found in $20 \%$ of ET patients in a study. Hence, aVWD should be assessed in those with a platelet count of $>1$ million/cmm especially before starting aspirin [41].

\section{Cytoreductive Therapy}

Apart from aspirin, cytoreductive therapy is indicated to prevent thrombotic complications in high-risk categories. The usual treatment goal with cytoreductive therapies is a platelet count between 100,000 and $400,000 / \mathrm{cmm}$ [20]. Hydroxyurea (HU) is preferred as it has been proven to reduce the risk of thrombosis in randomized control trials in high-risk patients comparing $\mathrm{HU}$ versus no myelosuppressive therapy [50,51]. Given its gonadal toxicity, mutagenicity, and teratogenic effects, HU is usually avoided in young individuals, women of childbearing age, and pregnant patients. Noninferiority of anagrelide in comparison with HU has been established; however, it is associated with adverse effects like myelofibrosis and cardiac events $[32,33]$. Busulfan, interferon, and ruxolitinib are also used as alternative cytoreductive therapies $[20,52,53]$.

\section{Early Management of Acute Ischemic Stroke in ET}

In the following few paragraphs, we summarize the current evidence of early management of acute ischemic stroke, mainly antithrombotic management. Further, we present the differences in antithrombotic therapy in different types of ischemic strokes, especially in the context of ET.
Ischemic Stroke within Revascularization Window

There is no separate recommendation for acute management of disabling ischemic stroke in patients with ET when they present within revascularization time window for thrombolysis or endovascular thrombectomy [54].

Antiplatelet Therapy Initiation after Acute

Atherothrombotic Ischemic Stroke in ET

In ischemic stroke, antiplatelet is generally recommended within 24-48 h after the onset of stroke. However, in cases of ischemic stroke with ET, ideally aVWD should be ruled out before starting ASA if the platelet count is $>1,000,000-1,500,000 / \mathrm{cmm}$ for the reasons mentioned above $[55,56]$. There are a few indications of dual antiplatelet therapy (DAPT) in ischemic stroke and TIAs. DAPT is generally recommended for 3 weeks for minor noncardioembolic ischemic stroke (NIHSS score $\leq 3$ ) and TIAs who did not receive thrombolysis and for 3 months for ischemic stroke or TIA attributable to severe stenosis (70-99\%) of a major intracranial artery. After this duration, the single antiplatelet agent should be continued lifelong for secondary stroke prevention $[54,57,58]$. Given inadequate suppression of platelets in ET with recommended dose/dosing schedule of low-dose antiplatelet for $\mathrm{CV}$ protection, experts suggest low-dose ASA twice daily in arterial thrombotic diseases if they have additional risk factors like age $>60$ years or JAK 2 mutation or presence of CV risk factors [39]. Taking into account both these considerations, ET patients presenting with noncardioembolic stroke/TIA with either of the abovementioned risk factors should be managed with DAPT for 3 weeks-3 months followed by consideration of low-dose ASA twice daily for reducing recurrent ischemic strokes.

\section{Antithrombotic Management of Cardioembolic}

Stroke in ET

Generally, oral anticoagulant therapy is indicated within 14 days of onset of cardioembolic stroke attributable to AF, for secondary stroke prevention. However, whether the additional antiplatelet agent should be included in their antithrombotic management in ET patients in presence of additional risk factors (age $>60$ years or JAK 2 mutation or presence of CV risk factors) is an area of uncertainty.

Antithrombotic Management of Stroke Secondary to Cerebral Venous Thrombosis in ET

Expert recommendations suggest simultaneous lowdose ASA once daily and anticoagulant use in venous thrombotic disease including cerebral venous thrombosis 
in ET patients with additional risk factors as mentioned before [43]. However, these management decisions may not be easy and straightforward given increased risk of bleeding with dual antithrombotic agents.

\section{Risk-Benefit Balance of Concurrent Antiplatelet and}

Anticoagulant Antithrombotic Management

Currently, the recommendation for concurrent antiplatelet and anticoagulant antithrombotic management is for patients with $\mathrm{AF}$ at increased risk of stroke and simultaneous coronary or peripheral artery disease requiring stenting or simultaneous presentation of acute coronary syndrome [57]. Some of these patients require even concurrent triple therapy with DAPT and anticoagulant depending on indications like acute coronary syndrome with percutaneous coronary intervention or elective percutaneous coronary intervention with high-risk features for thrombotic CV events $[59,60]$. These simultaneous dual or triple antithrombotics in AF with CAD are based on the findings of PIONEER AF-PCI, RE-DUAL PCI, and AUGUSTUS trials [61-63]. The duration of concurrent antiplatelet and anticoagulant therapy in these recommendations varies based on balanced assessment of their risk of thromboembolic event and clinically relevant bleeding associated with the use of antithrombotic agents $[59,64]$. Risk calculators like Stroke Prevention in Atrial Fibrillation Risk Calculator (SPARC), $\mathrm{HEMORR}_{2} \mathrm{HAGES}$ Score, HAS-BLED Score, ATRIA Bleeding Risk Score, CHADS2, and CHADS2-Vasc scores provide a quantitative assessment of bleeding with the use of antithrombotic/anticoagulants and thrombotic risk with AF useful for patient-centered decision-making [65-70]. Furthermore, the involvement of multidisciplinary teams like Hematology, Thromboembolism, and Neurology/Stroke services would be useful in these complex scenarios for management choices.

\section{Conclusion}

Given high risk of thrombosis, ET patients are at increased risk of ischemic stroke/TIA, resulting from both cerebral arterial and venous thrombosis. Early management of acute ischemic stroke in ET patients presenting within the revascularization time window does not differ from non-ET patients. ET patients with ischemic cerebrovascular events fall into the high-risk category for thrombotic events. Thus, the expert consensus is to treat such patients with low-dose aspirin twice daily instead of once daily if they have concomitant risk factors such as age $>60$ years, JAK2 mutation, and presence of CV risk factors. However, antithrombotic management could be challenging in patients with cerebral venous thrombosis or cardioembolic stroke in presence of these additional risk factors, as there is uncertainty about the benefit of adding antiplatelet agents on the top of anticoagulant therapy in such patients. In the absence of high-quality evidence, more research is needed to address these dilemmas.

\section{Statement of Ethics}

Written informed consent was obtained from the patient presented in the manuscript.

\section{Conflict of Interest Statement}

The authors have no conflicts of interest to declare.

\section{Funding Sources}

No grants were received for the preparation of the present manuscript.

\section{Author Contributions}

S.D.: design, literature search, organization, and preparation of the manuscript; A.D.: revision and preparation of the final draft of the manuscript. T.P.: case report. The final version of the manuscript was approved by S.D., A.D., and T.P.

\section{References}

1 Mehta J, Wang H, Iqbal SU, Mesa R. Epidemiology of myeloproliferative neoplasms in the United States. Leuk Lymphoma. 2014; 55(55):595-600.

2 Tefferi A. Myeloproliferative neoplasms: a decade of discoveries and treatment advances. Am J Hematol. 2016;91(1):50-8.

3 Tefferi A, Pardanani A. Myeloproliferative neoplasms: a contemporary review. JAMA Oncol. 2015;1(1):97-105.

4 Schafer AI. Thrombocytosis. N Engl J Med. 2004;350(12):1211-9.

5 Arber DA, Orazi A, Hasserjian R, Thiele J, Borowitz MJ, Le Beau MM, et al. The 2016 revision to the World Health Organization classification of myeloid neoplasms and acute leukemia. Blood. 2016;127(20):2391-405.

6 Tefferi A, Guglielmelli P, Larson DR, Finke C, Wassie EA, Pieri L, et al. Long-term survival and blast transformation in molecularly annotated essential thrombocythemia, polycythemia vera, and myelofibrosis. Blood. 2014; 124(16):2507-615. 
7 Cerquozzi S, Tefferi A. Blast transformation and fibrotic progression in polycythemia vera and essential thrombocythemia: a literature review of incidence and risk factors. Blood Cancer J. 2015;5(5):e366.

8 Barosi G, Mesa R, Finazzi G, Harrison C, Kiladjian JJ, Lengfelder E, et al. Revised response criteria for polycythemia vera and essential thrombocythemia: an ELN and IWG-MRT consensus project. Blood. 2013;121(23):4778-81.

9 Tefferi A, Fonseca R, Pereira DL, Hoagland HC. A long-term retrospective study of young women with essential thrombocythemia. Mayo Clin Proc. 2001;76(76):22-8.

10 Fenaux P, Simon M, Caulier MT, Lai JL, Goudemand J, Bauters F. Clinical course of essential thrombocythemia in 147 cases. Cancer. 1990;66(3):549-56.

11 Wolanskyj AP, Lasho TL, Schwager SM, McClure RF, Wadleigh M, Lee SJ, et al. JAK2 mutation in essential thrombocythaemia: clinical associations and long-term prognostic relevance. Br J Haematol. 2005;131(131):208-13.

12 Chistolini A, Mazzucconi MG, Ferrari A, La Verde G, Ferrazza G, Dragoni F, et al. Essential thrombocythemia: a retrospective study on the clinical course of 100 patients. Haematologica. 1990;75(6):537-40.

13 Cortelazzo S, Viero P, Finazzi G, D’Emilio A, Rodeghiero F, Barbui T. Incidence and risk factors for thrombotic complications in a historical cohort of 100 patients with essential thrombocythemia. J Clin Oncol. 1990;8(8): 556-62.

14 Bellucci S, Janvier M, Tobelem G, Flandrin G, Charpak Y, Berger R, et al. Essential thrombocythemias. Clinical evolutionary and biological data. Cancer. 1986;58(11):2440-7.

15 Colombi M, Radaelli F, Zocchi L, Maiolo AT Thrombotic and hemorrhagic complications in essential thrombocythemia. A retrospective study of 103 patients. Cancer. 1991; 67(67):2926-30.

16 Martinelli I, De Stefano V, Carobbio A, Randi ML, Santarossa C, Rambaldi A, et al. Cerebral vein thrombosis in patients with Philadelphia-negative myeloproliferative neoplasms. An European Leukemia Net study. Am J Hematol. 2014;89(11):E200-5.

17 Kato Y, Hayashi T, Sehara Y, Deguchi I, Fukuoka T, Maruyama $\mathrm{H}$, et al. Ischemic stroke with essential thrombocythemia: a case series. I Stroke Cerebrovasc Dis. 2015;24(24):890-3.

18 Posfai E, Marton I, Szoke A, Borbenyi Z, Vecsei L, Csomor A, et al. Stroke in essential thrombocythemia. J Neurol Sci. 2014;336(12):260-2.

19 Richard S, Perrin J, Baillot PA, Lacour JC, Ducrocq X. Ischaemic stroke and essential thrombocythemia: a series of 14 cases. Eur J Neurol. 2011;18(18):995-8.

20 Barbui T, Barosi G, Birgegard G, Cervantes F, Finazzi G, Griesshammer M, et al. Philadelphia-negative classical myeloproliferative neoplasms: critical concepts and management recommendations from European LeukemiaNet. J Clin Oncol. 2011;29(6):761-70.
21 Barbui T, Finazzi G, Carobbio A, Thiele J, Passamonti F, Rumi E, et al. Development and validation of an International Prognostic Score of thrombosis in World Health Organization-essential thrombocythemia (IPSETthrombosis). Blood. 2012;120(120):5128252.

22 Barbui T. International prognostic score for thrombosis in essential thrombocythemia (ET) calculator. Available from: https://www. siematologia.it/LG/IPSET\%20thrombosi/ IPSET\%20thrombosihtm. Accessed $7 \mathrm{Feb}$ 2021.

23 Barbui T, Vannucchi AM, Buxhofer-Ausch V, De Stefano V, Betti S, Rambaldi A, et al. Practice-relevant revision of IPSET-thrombosis based on 1019 patients with WHO-defined essential thrombocythemia. Blood Can cer J. 2015;5:e369.

24 Haider M, Gangat N, Lasho T, Abou Hussein AK, Elala YC, Hanson C, et al. Validation of the revised international prognostic score of thrombosis for essential thrombocythemia (IPSET-thrombosis) in 585 Mayo clinic patients. Am J Hematol. 2016;91(91): 390-4.

25 Carobbio A, Thiele J, Passamonti F, Rumi E, Ruggeri M, Rodeghiero F, et al. Risk factors for arterial and venous thrombosis in WHOdefined essential thrombocythemia: an international study of 891 patients. Blood. 2011; 117(22):5857-9.

26 Carobbio A, Finazzi G, Guerini V, Spinelli O, Delaini F, Marchioli R, et al. Leukocytosis is a risk factor for thrombosis in essential thrombocythemia: interaction with treatment, standard risk factors, and Jak2 mutation status. Blood. 2007;109(109):2310-3.

27 Palandri F, Polverelli N, Catani L, Ottaviani E, Baccarani M, Vianelli N. Impact of leukocytosis on thrombotic risk and survival in 532 patients with essential thrombocythemia: a retrospective study. Ann Hematol. 2011; 90(8):933-8.

28 Landolfi R, Di Gennaro L, Barbui T, De Stefano V, Finazzi G, Marfisi R, et al. Leukocytosis as a major thrombotic risk factor in patients with polycythemia vera. Blood. 2007; 109(109):2446-52.

29 Gangat N, Wolanskyj AP, Schwager SM, Hanson CA, Tefferi A. Leukocytosis at diagnosis and the risk of subsequent thrombosis in patients with low-risk essential thrombocythemia and polycythemia vera. Cancer. 2009; 115(24):5740-5.

30 Girodon F, Dutrillaux F, Broséus J, Mounier M, Goussot V, Bardonnaud P, et al. Leukocytosis is associated with poor survival but not with increased risk of thrombosis in essential thrombocythemia: a population-based study of 311 patients. Leukemia. 2010;24(24):9003.

31 Carobbio A, Ferrari A, Masciulli A, Ghirardi A, Barosi G, Barbui T. Leukocytosis and thrombosis in essential thrombocythemia and polycythemia vera: a systematic review and meta-analysis. Blood Adv. 2019;3(3):1729-37.
32 Gisslinger H, Gotic M, Holowiecki J, Penka M, Thiele J, Kvasnicka HM, et al. Anagrelide compared with hydroxyurea in WHO-classified essential thrombocythemia: the ANAHYDRET Study, a randomized controlled trial. Blood. 2013;121(10):1720-8.

33 Harrison CN, Campbell PJ, Buck G, Wheatley K, East CL, Bareford D, et al. Hydroxyurea compared with anagrelide in high-risk essential thrombocythemia. N Engl J Med. 2005; 353(353):33-45.

34 Klampfl T, Gisslinger H, Harutyunyan AS, Nivarthi H, Rumi E, Milosevic JD, et al. Somatic mutations of calreticulin in myeloproliferative neoplasms. N Engl J Med. 2013; 369(25):2379-90

35 Nangalia J, Massie CE, Baxter EJ, Nice FL, Gundem G, Wedge DC, et al. Somatic CALR mutations in myeloproliferative neoplasms with nonmutated JAK2. N Engl J Med. 2013; 369(369):2391-405.

36 Finazzi G, Carobbio A, Guglielmelli P, Cavalloni C, Salmoiraghi S, Vannucchi AM, et al. Calreticulin mutation does not modify the IPSET score for predicting the risk of thrombosis among 1150 patients with essential thrombocythemia. Blood. 2014;124(16):2611-2.

37 Rotunno G, Mannarelli C, Guglielmelli P, Pacilli A, Pancrazzi A, Pieri L, et al. Impact of calreticulin mutations on clinical and hematological phenotype and outcome in essential thrombocythemia. Blood. 2014;123(123):1552-5.

38 Hauschner H, Bokstad Horev M, Misgav M, Nagar M, Seligsohn U, Rosenberg N, et al. Platelets from calreticulin mutated essential thrombocythemia patients are less reactive than JAK2 V617F mutated platelets. Am J Hematol. 2020;95(4):379-86.

39 Barbui T. Refining prognostication of thrombosis in ET. Am J Hematol. 2016;91(4):361-3.

40 Latagliata R, Montanaro M, Cedrone M, Di Veroli A, Spirito F, Santoro C, et al. High platelet count at diagnosis is a protective factor for thrombosis in patients with essential thrombocythemia. Thromb Res. 2017; 156(156):168-71.

41 Mital A, Prejzner W, Bieniaszewska M, Hellmann A. Prevalence of acquired von Willebrand syndrome during essential thrombocythemia: a retrospective analysis of 170 consecutive patients. Pol Arch Med Wewn. 2015; 125(12):914-20.

42 Mesa RA, Jamieson C, Bhatia R, Deininger MW, Fletcher CD, Gerds AT, et al. NCCN guidelines insights: myeloproliferative neoplasms, version 2.2018. J Natl Compr Canc Netw. 2017;15(15):1193-207.

43 Tefferi A, Vannucchi AM, Barbui T. Essential thrombocythemia treatment algorithm 2018. Blood Cancer J. 2018;8(8):2.

44 Landolfi R, Marchioli R, Kutti J. Efficacy and safety of low-dose aspirin in polycythemia vera. N Engl J Med. 2004;350(2):114-24.

45 Marchioli R, Finazzi G, Specchia G. Cardiovascular events and intensity of treatment in polycythemia vera. N Engl J Med. 2013; 368(368):22-33. 
46 Alvarez-Larrán A, Cervantes F, Pereira A, Arellano-Rodrigo E, Pérez-Andreu V, Hernández-Boluda $\mathrm{J}-\mathrm{C}$, et al. Observation versus antiplatelet therapy as primary prophylaxis for thrombosis in low-risk essential thrombocythemia. Blood. 2010;116(8):1205387.

47 Pascale S, Petrucci G, Dragani A, Habib A, Zaccardi F, Pagliaccia F, et al. Aspirin-insensitive thromboxane biosynthesis in essential thrombocythemia is explained by accelerated renewal of the drug target. Blood. 2012; 119(119):3595-603.

48 Dragani A, Pascale S, Recchiuti A, Mattoscio D, Lattanzio S, Petrucci G, et al. The contribution of cyclooxygenase- 1 and -2 to persistent thromboxane biosynthesis in aspirin-treated essential thrombocythemia: implications for antiplatelet therapy. Blood. 2010;115(5): 1054-61.

49 Dillinger JG, Sideris G, Henry P, Bal dit Sollier C, Ronez E, Drouet L. Twice daily aspirin to improve biological aspirin efficacy in patients with essential thrombocythemia. Thromb Res. 2012;129(129):91-4.

50 Cortelazzo S, Finazzi G, Ruggeri M, Vestri O, Galli M, Rodeghiero F, et al. Hydroxyurea for patients with essential thrombocythemia and a high risk of thrombosis. N Engl J Med. 1995; 332(17):1132-6.

51 Finazzi G, Ruggeri M, Rodeghiero F, Barbui T. Second malignancies in patients with essential thrombocythaemia treated with busulphan and hydroxyurea: long-term follow-up of a randomized clinical trial. Br J Haematol. 2000;110(110):577-83.

52 Yacoub A, Mascarenhas J, Kosiorek H, Prchal JT, Berenzon D, Baer MR, et al. Pegylated interferon alfa-2a for polycythemia vera or essential thrombocythemia resistant or intolerant to hydroxyurea. Blood. 2019;134(134): 1498-509.

53 Harrison CN, Mead AJ, Panchal A, Fox S, Yap C, Gbandi E, et al. Ruxolitinib vs best available therapy for ET intolerant or resistant to hydroxycarbamide. Blood. 2017;130(17):188997.
54 Powers WJ, Rabinstein AA, Ackerson T, Adeoye OM, Bambakidis NC, Becker K, et al. Guidelines for the early management of patients with acute ischemic stroke: 2019 update to the 2018 guidelines for the early management of acute ischemic stroke: a guideline for healthcare professionals from the American Heart Association/American Stroke Association. Stroke. 2019;50(50):e344-418.

55 Awada H, Voso MT, Guglielmelli P, Gurnari C. Essential thrombocythemia and acquired von Willebrand syndrome: the shadowlands between thrombosis and bleeding. Cancers. 2020;12(12): 1746 .

56 Kreher S, Ochsenreither S, Trappe RU, Pabinger I, Bergmann F, Petrides PE, et al. Prophylaxis and management of venous thromboembolism in patients with myeloproliferative neoplasms: consensus statement of the Haemostasis Working Party of the German Society of Hematology and Oncology (DGHO), the Austrian Society of Hematology and Oncology (ÖGHO) and Society of Thrombosis and Haemostasis Research (GTH e.V.). Ann Hematol. 2014;93(12):1953-63.

57 Kernan WN, Ovbiagele B, Black HR, Bravata DM, Chimowitz MI, Ezekowitz MD, et al. Guidelines for the prevention of stroke in patients with stroke and transient ischemic attack: a guideline for healthcare professionals from the American Heart Association/American Stroke Association. Stroke. 2014;45(45): 2160-236.

58 CanadianStrokeBestPractices. Canadian Stroke Best Practices Guideline. Available from: https: //www.strokebestpractices.ca/ recommendations. Accessed $2021 \mathrm{Feb} 7$.

59 January CT, Wann LS, Calkins H, Chen LY, Cigarroa JE, Cleveland JC Jr, et al. 2019 AHA/ ACC/HRS focused update of the 2014 AHA/ ACC/HRS guideline for the management of patients with atrial fibrillation: a report of the American College of Cardiology/American Heart Association Task Force on clinical practice guidelines and the heart rhythm society in collaboration with the society of thoracic surgeons. Circulation. 2019;140(140): e125-e51.

60 Andrade JG, Aguilar M, Atzema C, Bell A, Cairns JA, Cheung CC, et al. The 2020 Canadian Cardiovascular Society/Canadian Heart Rhythm Society comprehensive guidelines for the management of atrial fibrillation. Can J Cardiol. 2020;36(12):1847-948.
61 Gibson CM, Mehran R, Bode C, Halperin J, Verheugt FW, Wildgoose $\mathrm{P}$, et al. Prevention of bleeding in patients with atrial fibrillation undergoing PCI. N Engl J Med. 2016;375(25): 2423-34.

62 Cannon CP, Bhatt DL, Oldgren J, Lip GYH Ellis SG, Kimura T, et al. Dual antithrombotic therapy with dabigatran after PCI in atrial fibrillation. N Engl J Med. 2017;377(377): 1513-24.

63 Lopes RD, Heizer G, Aronson R, Vora AN, Massaro T, Mehran R, et al. Antithrombotic therapy after acute coronary syndrome or PCI in atrial fibrillation. N Engl J Med. 2019; 380(380):1509-24.

64 Mehta SR, Bainey KR, Cantor WJ, Lordkipanidzé M, Marquis-Gravel G, Robinson SD, et al. 2018 Canadian Cardiovascular Society/Canadian Association of Interventional Cardiology focused update of the guidelines for the use of antiplatelet therapy. Can J Cardiol. 2018;34(34):214-33.

65 Loewen P. SPARC: stroke prevention in atrial fibrillation risk tool version 9. 2019 May. Available from: https://www.sparctool.com/.

66 Gage B. HEMORR2HAGES score for major bleeding risk. Available from: https://www. $\mathrm{mdcalc}$.com/hemorr2hages-score-majorbleeding-risk\# $\sim$ text $=$ The $\% 20 \mathrm{HEMOR}$ R2HAGES\%20 score $\% 20$ was $\% 20$ developed $\% 20$ to $\% 20$ quantify $\% 20$ the, the $\% 20$ HEMORR2HAGES\%20mnemonic. Accessed 2021 Feb 7.

67 Pisters R. HAS-BLED score for major bleeding risk. Available from: https://www.mdcalc. com/has-bled-score-major-bleeding-risk. Accessed 2021 Feb 7.

68 Singer D. ATRIA bleeding risk score. Available from: https://www.mdcalc.com/atriableeding-risk-score. Accessed $2021 \mathrm{Feb} 7$.

69 Gage B. CHADS2 score for atrial fibrillation stroke risk. Available from: https://www.mdcalc.com/chads2-score-atrial-fibrillationstroke-risk. Accessed $2021 \mathrm{Feb} 7$.

70 Lip G. CHA2DS2-VASc score for atrial fibrillation stroke risk. Available from: https:// www.mdcalc.com/cha2ds2-vasc-score-atrialfibrillation-stroke-risk\#why-use. Accessed 2021 Feb 7. 